Relations industrielles

Industrial Relations

\title{
Sennett, Richard, Le travail sans qualités : les conséquences humaines de la flexibilité
}

\section{Fernand Morin}

Volume 55, numéro 4, 2000

URI : https://id.erudit.org/iderudit/051368ar

DOI : https://doi.org/10.7202/051368ar

Aller au sommaire du numéro

Éditeur(s)

Département des relations industrielles de l'Université Laval

ISSN

0034-379X (imprimé)

1703-8138 (numérique)

Découvrir la revue

Citer ce compte rendu

Morin, F. (2000). Compte rendu de [Sennett, Richard, Le travail sans qualités : les conséquences humaines de la flexibilité]. Relations industrielles / Industrial Relations, 55(4), 793-795. https://doi.org/10.7202/051368ar

Tous droits réservés (C Département des relations industrielles de l'Université Laval, 2000
Ce document est protégé par la loi sur le droit d'auteur. L’utilisation des services d'Érudit (y compris la reproduction) est assujettie à sa politique d'utilisation que vous pouvez consulter en ligne.

https://apropos.erudit.org/fr/usagers/politique-dutilisation/ 
workers on the eve of their victory: "Ravenswood is the place where working class people said we're going to push until we push you back across the river. It's a place where workers are putting
Corporate America on notice that they can't rob us of our dignity."

Margaret Hallock

University of Oregon

\section{Le travail sans qualités : les conséquences humaines de la flexibilité par Richard SENNETT, traduit de l'américain par Pierre Emmanuel DAUZAT, Paris : Albin Michel, 2000, 223 p., ISBN 2-226-11501-3.}

Il s'agit de la traduction de l'ouvrage paru en 1998 sous le titre The Corrosion of Character: The Personal Consequences of Work in the New Capitalism, New York : W.W. Norton \& Company.

L'auteur nous fait part de ses constats, observations et réflexions au sujet de certains effets du travail en cette nouvelle économie. À cette fin, le professeur Sennett rappelle le sens étymologique de quelques termes autrefois chéris tels que carrière, caractère, job, etc. pour nous démontrer par la suite comment ils ont perdu leur sens traditionnel. En effet, nous dit-il, comment pouvons-nous faire carrière, soit suivre une voie dans une direction souhaitée, en un monde où seul l'immédiat semble s'imposer. Comment le travailleur peut-il développer et affirmer son caractère alors que les fonctions successives qu'impose la flexibilité le privent de tout repère ? Cette flexibilité, rappelle-t-il, n'a plus le sens qu'on lui donnait, à savoir ployer face à la secousse puis reprendre sa forme originale alors que le travailleur dit "flexible» ploie sous la contrainte et se déplace pour tenter de devenir un automate " polyfonctionnel ».

Il ne s'agit pas d'une étude théorique où l'on construit une savante démonstration à l'aide de quelques modèles et notamment par voie déductive. Le professeur Sennett fait appel à quelques situations bien concrètes et relativement simples pour étayer ses propos. Ainsi, le lecteur peut facilement partager ce discours tellement, sans être aussi un féru de la sociologie du travail, il connaît ou reconnaît lui-même de sem- blables situations de fait qui meublent son voisinage ou qui hantent son esprit. Dès lors, les observations fort perspicaces de l'auteur vont être plus facilement retenues et comprises. Au strict plan pédagogique, une telle approche rend le livre plus accessible à un vaste public.

On ne saurait cependant considérer cet ouvrage comme une simple vulgarisation de l'état du monde du travail sous le soleil néocapitaliste. L'auteur prend habilement à témoin les ouvrages les plus classiques tels que ceux de Virgile, Rousseau, Locke, Smith, Voltaire, Weber, Lippman, etc. De plus, l'ouvrage comprend, en annexes, dix tableaux statistiques susceptibles de mieux illustrer ses thèses.

L'auteur retient particulièrement trois lieux de travail qui connurent des transformations radicales quoique fort différentes les unes des autres. Le premier cas, et sans doute le principal, vise une boulangerie qu'il visita d'abord à l'occasion d'études de terrain en vue de son ouvrage Les tyrannies de l'intimité paru il y a vingt-cinq ans. Cette boulangerie italienne de Boston, où les boulangers étaient grecs, subsiste encore mais comme simple fond de commerce maintenant qu'elle est intégrée par voie d'acquisition à une entreprise d'envergure nationale. Par la magie de l'informatique, on ne goûte plus la pâte, on ne la palpe plus, on ne la sent plus, on ne fait que la regarder in vitro et la manipuler à l'aide des icônes du tableau de bord qui, en fonction du logiciel, normalisent les processus de production (programme SIMS). La chaleur ambiante, la sueur 
des boulangers, les parfums qui s'entrechoquent, l'air parfois raréfié, sont des choses du passé. Certes, le port du sarrau blanc est de mise, mais au-delà de l'uniforme symbolique, s' agit-il encore de boulangers? Sennett souligne deux observations qui permettent de bien comprendre la portée de la métamorphose de cette activité professionnelle où, en bout de ligne, on fabrique encore du pain :

— Ces « boulangers » refusent l'offre de formation professionnelle en boulangerie car, disent-ils, pourquoi apprendre ce métier puisque demain nous ferons autre chose (p. 94). Pour l'instant, il leur suffit de savoir presser sur les bons icônes en temps et selon l'ordre du programme élaboré par d'autres pour mettre en fonction une «machine compétente » par le truchement d'opérateurs indifférents. Ce travail « aveugle » rend le lieu aseptisé et sans convivialité (p.97). L'auteur en dégage cette observation sous forme d'équation : « la compréhension du travail est superficielle, son identité professionnelle est précaire » (p. 101).

- Une jeune dame postule à la boulangerie et le contremaître lui demande si elle dispose de quelques expériences professionnelles en boulangerie. Surprise d'une telle question, elle se contente de dire qu'elle connait bien ce type de logiciels et que le fait qu'elle pourrait produire un jour du pain et le lendemain, toute autre chose ne lui paraît pas important puisque la mécanique informatisée demeure l'étalon de l'ordre de production.

La deuxième illustration retenue par l'auteur porte sur la mésaventure de Rose qui délaissa son bistrot pour tenter sa chance dans une firme de publicité et de marketing. En prenant délibérément ce « risque ", c'est-à-dire oser sans cesse rempoter une plante pourtant en pleine croissance (p. 110), elle ne pouvait que connaître les avatars de ce monde superficiel sans passé, sans avenir et où tout se conjugue au présent : « la flèche du temps n'a plus de trajectoire » (p. 137). En ces milieux, ne rien faire est vu comme une passivité et nullement comme acte de prudence.

Le troisième exemple comprend un groupe de gestionnaires subrepticement mis à pied par IBM. Ces ex-collègues de travail se rencontrent et discutent des faits immédiats qu'ils retiennent à titre de causes possibles de leurs déboires. Il leur fallut beaucoup de temps à saisir ou à pouvoir reconnaître les facteurs de fond qui permettent de comprendre leur déconfiture (p. 126). En somme, nous dit Sennett, ils étaient prisonniers du présent et semblables au lapin « qui ne quitte pas des yeux les pattes du renard ». C'est à croire que les forces du destin expliqueraient tout et que le travailleur deviendrait simple objet de la contingence. Pourtant, souligne-t-il, il est vrai que le domaine du risque s'étend démesurément, mais la répartition des profits ne semble pas répondre de la même logique.

S'inspirant des concepts lean et mean de Bennett Harrison, l'auteur situe ainsi les détenteurs du pouvoir en ce néocapitalisme : « la grande société tient sous sa coupe le corps de ballet de ses soustraitants, se défaussant sur ses partenaires plus faibles, plus durement touchés en cas de récession ou d'échec du produit. Les îlots de travail demeurent au large du continent du pouvoir » (p. 75). C'est ainsi que les familles dites de la classe moyenne (catégorie plus économique que sociale) auraient besoin de deux salaires pour garder le cap.

Richard Sennett nous offre aussi de fructueuses observations d'ordre éthique sur le sens donné au travail à travers les siècles. Ses réflexions sont particulièrement intéressantes alors que nous connaissons une nette distanciation, sinon rupture, entre le sujet et l'objet du travail (p. 137 à 166). En quelques pages seulement, l'auteur rappelle les grandes étapes franchies afin de savoir comment « bâtir sa vie ", l'homo faber. S'inspirant des Georgiques de Virgile, il reconnaît, à 
l'instar du paysan en lutte avec la terre, que nous disposons de la capacité d'œuvrer à notre perte en ce combat anarchique : "Contre ces tempêtes intérieures, la seule défense de l'individu est de bien organiser son temps » (p. 141). Puis, à l'aide de Max Weber, il explique comment l'homme accepta de travailler à la sueur de son front dans l'espoir d'une gratification différée. L'éthique protestante incita à l'épargne et nullement à la consommation et par le travail, l'homme devait pouvoir prouver aux autres et à l'Autre sa valeur morale (p. 147).

Le véritable travail en équipe devrait favoriser des attentions mutuelles plutôt que l'automutilation individuelle. Sennett souligne qu'il n'en est pas ainsi parce qu'il ne s'agit vraiment pas d'un travail d'équipe mais de simples regroupements superficiels où l'acte de communication importe davantage que les faits communiqués. Il conclut alors en termes quelque peu alarmistes ce passage au néocapitalisme : «Quittant le champ de la tragédie, les relations humaines tournent à la farce » (p. 149).

Finalement, Richard Sennett explique que l'action incontinente du néocapitalisme ne pourrait être contenue que lorsque l'on saura répondre raisonnablement à cette double question : «quelle valeur représente l'entreprise pour la collectivité, en quoi elle sert les intérêts civiques, plutôt que son seul registre des pertes et profits $»$ (p. 194). Nous devons reconnaître que la lecture de cet ouvrage, Le travail sans qualités ne pourrait qu'enrichir et stimuler la réflexion de l'étudiant en relations industrielles. De même en serait-il pour toute personne qui s'intéresse vraiment aux questions du travail et surtout, à la personne même du travailleur.

Fernand Morin Université Laval

\section{The Working Class Majority: America's Best Kept Secret}

by Michael ZWEIG, Ithaca, N.Y.: ILR Press, 2000, 198 pp., ISBN 0-8014$3637-0$.

TINA: "There is no alternative." Margaret Thatcher popularized the phrase 20 years ago, and conservatives throughout the world took it up. The genius of Thatcher and Reagan lay in their successful ability to mobilize the rich and the corporate elite to act consciously and more publicly in their own narrow interests. Increasingly the advocates of TINA were able to ally with sections of the middle class and project the image that they - not the working classes - were the majority.

Intellectuals also took up the cry. Hundreds of academic works appeared describing the folly of socialism, the negative effects of unions, and the debilitating effects of welfare. The research was buttressed by claims that the working class, which might have needed unions in the past, no longer existed or, if it did, it was a new working class different from the old.

Unfortunately for working people all over the world, their erstwhile allies also accepted the idea that the old structures of workers' power were no longer needed. Even more unfortunate, many labour leaders also seemed to believe the TINA theory. In the political world, and especially in the U.S. and Great Britain, the result was a sharp move to the right by the Democratic and Labour parties.

While there are many explanations for the decline of organized labour, one aspect of the decline must surely be a disintegration of belief. If people no longer believe that there is a working class with distinct interests, then they are demobilized. It is also important to note 